\title{
Thermal equation of state BWR for water and water steam
}

\author{
Magda Vestfálová ${ }^{1, a}$, Markéta Petříková ${ }^{1}$ \\ ${ }^{1}$ KEZ, TU Liberec, Studentská 1402/2, 46117 Liberec 1, Czech Republic
}

\begin{abstract}
The fundamental bases for the calculation of the thermodynamic properties of substances are the thermal equation of state and the dependence of some of the basic specific heat capacities on temperature. Any other thermodynamic properties of a substance can already be deduced from these relations. The aim of this paper is to design the simple thermal equation of state for water steam based on original BWR thermal equation of state, which describes the behavior of $\mathrm{H}_{2} \mathrm{O}$ in the gaseous phase in region of usual pressures and temperatures with sufficient accuracy.
\end{abstract}

\section{Introduction}

Water and water vapor plays an important role in many phenomena occurring in nature. They are also the customary working substances in various industrial fields (for example in engineering industry, chemistry field, food industry, ...). Therefore, their properties are explored, measured and tabulated in detail. [1] Research of properties of water and water steam began to organize on an international level since 1929 to provide reliable and consistent data on their properties. In 1972 there was founded the International Association for the Properties of Water and Steam - IAPWS which deals with verification and interpolation of experimental data and with expressing of the thermodynamic and transport properties of water and steam. In 1995, there was accepted Pruß - Wagner equation as the international formulation of thermodynamic properties of water and steam for scientific purposes IAPWS-95 [2]. The equation is in the form of Helmholtz dimensionless function depending on temperature and reduced density. The equation is valid for the liquid and gas in a wide range of temperatures and pressures. It contains a large number of constants. International Standards properties of water and steam for example [3] are based on Scientific formulation IAPWS-95.

In many cases, there is necessary to use some "simple" equation for calculation of thermodynamic properties of water steam. "Simple" form of thermal equation of state will be applicable only on a limited range of temperatures and pressures, and it will have less accuracy. The aim of this paper is to present, if possible, a "simple" thermal equation of state and to define its application area, where agreed accuracy is maintained.

In 1940 Manson Benedict, G. B. Webb and L. C. Rubin constructed "quite simple" so called original
Benedict - Webb - Rubin thermal equation of state (hereinafter BWR) [4].

$$
\begin{aligned}
& p=r T \rho+r T \rho^{2} B_{0}+r T \rho^{3} b-A_{0} \rho^{2}-a \rho^{3}+ \\
& +a \alpha \rho^{6}-\frac{\rho^{2} C_{0}}{T^{2}}+\frac{c \rho^{3}}{T^{2}}\left(1+\gamma \rho^{2}\right) e^{-\gamma \rho^{2}} .
\end{aligned}
$$

Equation has some physical basics in the state behavior of gases. It contains an addition to the specific constant $r$ still eight parameters: $A_{0}, B_{0}, C_{0}, a, b, c, \alpha$ and $\gamma$, that characterize the individual properties of the gas. They must be determined from experimental dates by regression. So, as well as other multi-parameter equations, BWR equation can be only used to description of properties of substances for which there is sufficient amount of experimental data in wide range of pressures and temperatures. This requirement is fulfilled for water and water steam undoubtedly.

In 1973 modification of the Benedict-Webb-Rubin equation of state by Starling was made [5]

$$
\begin{aligned}
& p=r T \rho+r T \rho^{2} B_{0}+r T \rho^{3} b-A_{0} \rho^{2}- \\
& -a \rho^{3}+a \alpha \rho^{6}+\frac{\alpha d \rho^{6}}{T}-\frac{\rho^{2} C_{0}}{T^{2}}+ \\
& +\frac{\rho^{2} D_{0}}{T^{3}}+\frac{\rho^{2} E_{0}}{T^{4}}+\frac{c \rho^{3}}{T^{2}}\left(1+\gamma \rho^{2}\right) e^{-\gamma \rho^{2}} .
\end{aligned}
$$

Values of the various parameters for 15 substances can be found in work [5].

Another modification of the Benedict-Webb-Rubin equation of state was made in 1987 by Jacobsen and Stewart [6], it is noted as $\mathrm{mBWR}$

$$
p=\sum_{n=1}^{9} a_{n} \rho^{n}+\sum_{n=10}^{15} a_{n} \rho^{2 n-17} e^{-\gamma \rho^{2}} .
$$

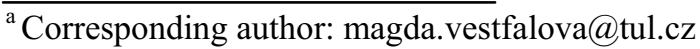


The mBWR equation subsequently evolved into a 32 term version with numerical parameters determined by fitting the equation to empirical data for a reference fluid [7].

At first BWR equation was suggested for calculation of behavior of hydrocarbons. For these substances values obtained from BWR equation are very close to the experimental date [8]. For these substances BWR equation describes the behavior of materials in single phase areas (it is in superheated steam and in the super cooled liquid) very well. In heterogeneous areas (below the limit curve), as expected, there is a local maximum and a local minimum and the results are totally unrealistic. Parameters of BWR equation for hydrocarbons are presented for example in work [9] too.

We want to obtain a "simple" equation of state for water and water steam, so we try to use the original BWR equation.

In [10] there was shown the using of BWR thermal equation of state for $\mathrm{H}_{2} \mathrm{O}$. There was described derivation of the isochoric specific heat capacity and enthalpy from the thermal equation BWR. It has been shown that in the area where the error of $p-v-T$ values obtained from BWR thermal state equations is small, the values of enthalpy, calculated from the relation derived using equation BWR are sufficiently precise too. The disadvantage was that the application area (the area with small errors) is very small.

The aim of this paper is to provide the values of constants of BWR equation $A_{0}, B_{0}, C_{0}, a, b, c, \alpha$ and $\gamma$ so that this BWR equation describes with a "sufficient" accuracy the behavior of water seam, it means $\mathrm{H}_{2} \mathrm{O}$ in gas phase in commonly used ranges of pressures and temperatures, and if possible tills to limit curve. A "reasonable" accuracy we will consider the deviation of the values obtained from the solution BWR and tabulated data according to formulation for scientific purposes IAPWS-95 was less than $1 \%$.

\section{Model of ideal gas}

The first term on the right side of BWR equation (1) $(r \cdot T \cdot \rho)$, which is proportional to the first power of density, corresponds to the state equation of ideal gas. Other members, proportional to the higher powers of density, represent the correction on the real behavior of gas. Obviously, the higher is the density, the greater is the influence of correction terms on the calculated pressure value.

In the "bigger" distances from the limit curve, it means in a state of much diluted gases (at "high" temperatures and "low" pressures, it means the "big" specific volumes, respectively "small" densities) the behavior of real materials approaches to the behavior of ideal gases. Correction terms in the BWR equation with higher power of density is unlikely to be strongly applied. In the case of water vapor we can illustrate this fact on several isotherms: error to $1 \%$ is for temperature $1000^{\circ} \mathrm{C}$ to the pressure $15 \mathrm{MPa}$, for temperature $500^{\circ} \mathrm{C}$ to the pressure $1 \mathrm{MPa}$, for temperature $200^{\circ} \mathrm{C}$ to the pressure $0.1 \mathrm{MPa}$, for temperature $100^{\circ} \mathrm{C}$ to the pressure $0.05 \mathrm{MPa}$ (in Figure 1. - red line). For temperature $50^{\circ} \mathrm{C}$ is the pressure of saturated steam very low $-0.012351 \mathrm{MPa}$. In this point (on the limit curve) we obtain values with accuracy $0.4 \%$ by using the model of ideal gas. The accuracy to $1 \%$ is maintained on the limit curve until the temperature $80^{\circ} \mathrm{C}$. In triple point (temperature $0.01^{\circ} \mathrm{C}$, pressure $0.000611657 \mathrm{MPa}$ ), the state of saturated steam is calculated with accuracy $0.07 \%$ using the model of ideal gas.

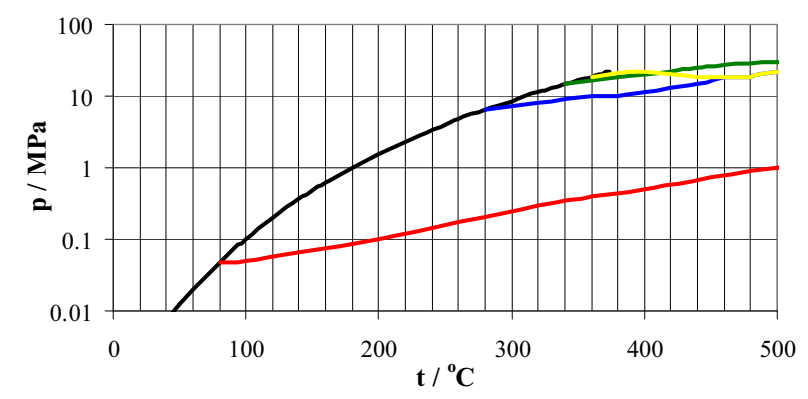

Figure 1. Field of application of the different models with the accuracy to $1 \%$ : thick line - the limit curve; red line - limit of applicability of ideal gas model; blue line - limit of applicability of model with correction $A_{0}, B_{0}$ and $C_{0}$; green line - limit of applicability of model with correction $A_{0}, B_{0}, C_{0}, a$ and $b$; yellow line - limit of application of BWR equation.

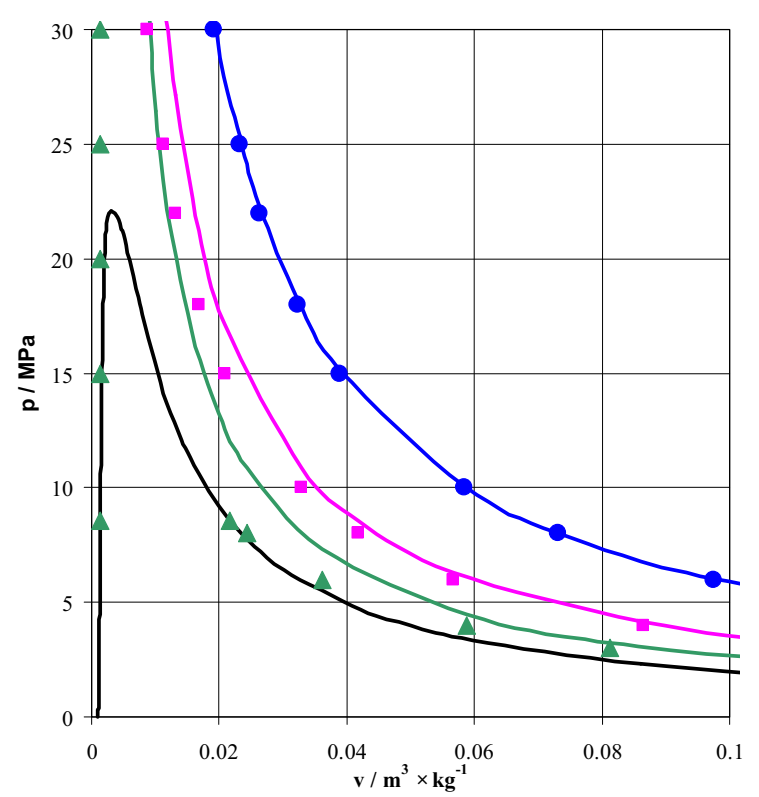

Figure 2. Comparison of isotherms of an ideal gas with real isotherms: thick line - the limit curve; green - isotherm $300^{\circ} \mathrm{C}$; pink - isotherm $500^{\circ} \mathrm{C}$; blue - isotherm $1000^{\circ} \mathrm{C}$; lines - ideal gas isotherms; points - real values.

Figure 2. shows the real isotherms $300^{\circ} \mathrm{C}, 500^{\circ} \mathrm{C}$ and $1000^{\circ} \mathrm{C}$ and corresponding isotherms calculated using the ideal gas model. With increasing specific volume is he ideal isotherms and the real isotherms close to each other, conversely a decreasing specific volume increases the difference between the ideal and the real isotherm. We obtain higher pressure using the model of ideal gas than its actual value at low specific volumes. 
Table 1. Specific volume $10^{3} \cdot \mathrm{v} / \mathrm{m}^{3} \mathrm{~kg}^{-1}$ of water steam depending on the pressure and temperature; yellow highlighted numbers accuracy of model of ideal gas less then $1 \%$; pink highlighted numbers - accuracy of model with correction $A_{0}, B_{0}$ and $C_{0}$ less then

$1 \%$; green highlighted numbers - accuracy of model with correction $A_{0}, B_{0}, C_{0}$, $a$ and $b$ less then $1 \%$.

\begin{tabular}{|c|c|c|c|c|c|c|c|c|c|c|c|}
\hline$t /{ }^{\circ} \mathrm{C} / \mathrm{MPa}$ & 18 & 15 & 10 & 8 & 6 & 4 & 2 & 1 & 0.5 & 0.1 & 0.01 \\
\hline 1000 & 32.282 & 38.808 & 58.390 & 73.079 & 97.560 & 146.52 & 293.42 & 587.21 & 1174.80 & 5875 & 58757 \\
\hline 500 & 16.810 & 20.827 & 32.811 & 41.767 & 56.671 & 86.44 & 175.68 & 354.11 & 710.94 & 3565 & 35679 \\
\hline 480 & 15.990 & 19.923 & 31.627 & 40.362 & 54.891 & 83.90 & 170.85 & 344.66 & 692.26 & 3473 & 34736 \\
\hline 460 & 15.116 & 18.973 & 30.407 & 38.926 & 53.083 & 81.34 & 165.98 & 335.19 & 673.56 & 3380 & 33833 \\
\hline 440 & 14.171 & 17.694 & 29.144 & 37.451 & 51.240 & 78.74 & 161.09 & 325.69 & 654.84 & 3288 & 32909 \\
\hline 420 & 13.122 & 16.874 & 27.826 & 35.928 & 49.355 & 76.11 & 156.17 & 316.17 & 636.09 & 3195 & 31986 \\
\hline 400 & 11.915 & 15.671 & 26.436 & 34.344 & 47.419 & 73.43 & 151.21 & 306.61 & 617.30 & 3103 & 31063 \\
\hline 380 & 10.419 & 14.289 & 24.950 & 32.681 & 45.418 & 70.70 & 146.21 & 297.00 & 598.47 & 3010 & 30139 \\
\hline 360 & 8.111 & 12.582 & 23.325 & 30.912 & 43.333 & 67.90 & 141.15 & 287.35 & 579.60 & 2917 & 29216 \\
\hline 340 & & & 21.487 & 28.992 & 41.135 & 65.02 & 136.03 & 277.64 & 560.67 & 2824 & 28292 \\
\hline 320 & & & 19.270 & 26.840 & 38.780 & 62.02 & 130.82 & 267.86 & 541.68 & 2732 & 27369 \\
\hline 300 & & & & 24.279 & 36.189 & 58.87 & 125.51 & 257.98 & 522.61 & 2639 & 26445 \\
\hline 280 & & & & & 33.199 & 55.50 & 120.05 & 248.00 & 503.42 & 2546 & 25521 \\
\hline 260 & & & & & & 51.78 & 114.41 & 237.88 & 484.12 & 2453 & 24598 \\
\hline 240 & & & & & & & 108.50 & 227.30 & 464.66 & 2360 & 23674 \\
\hline 220 & & & & & & & 102.18 & 216.98 & 444.96 & 2266 & 22749 \\
\hline 200 & & & & & & & & 206.02 & 425.03 & 2172 & 21825 \\
\hline 180 & & & & & & & & 194.44 & 404.63 & 2078 & 20901 \\
\hline 160 & & & & & & & & & 383.66 & 1984 & 19976 \\
\hline 140 & & & & & & & & & & 1889 & 19050 \\
\hline 120 & & & & & & & & & & 1793 & 18124 \\
\hline 100 & & & & & & & & & & 1696 & 17196 \\
\hline 50 & & & & & & & & & & & 14866 \\
\hline
\end{tabular}

In Table 1., the area in which the ideal gas model gives accuracy to $1 \%$ is "highlighted" in yellow.

\section{Correction using members proportional to the square of density}

If the state of gas comes "near" to the limit curve, it is (due to the independent variables in the BWR equation) if specific volume decreases (resp. density increases), the deviation of the ideal gas model from the fair value increases. In the BWR equation, the influence of the correction members with higher power density grows. The second, the fourth and the seventh term on the right side are proportional to the square of density (other members depend on even higher powers of density) their effects are thus manifested first. The fourth member $\left(-A_{0} \cdot \rho^{2}\right)$ is directly proportional only to the square of the density and it corresponds to the absolute deviation between the ideal and the real isotherm apparently. Whereas it is necessary to make such a correction, which "reduce" the ideal pressure in the BWR equation this member is subtracted. Deviation between ideal and real isotherm is not the same for all temperatures. At high temperatures (the substance is closer to the model of an ideal gas) the deviation is less, therefore the first correction (reduction of the ideal pressure due to fourth member) is "mitigated" due to the second member $\left(r \cdot T \cdot \rho^{2} \cdot B_{0}\right)$, which depends directly proportional to temperature, it is its influence increases with increasing of temperature. In the temperature range where the substance is describable due to ideal gas model, the fourth and the second member are mutually compensated. Conversely, at low temperatures, where the substance approaches the limit of liquefaction, is the absolute difference between the ideal and actual pressure greater correction from ideal behavior is reinforced due to seventh member - $\left(-\frac{\rho^{2} \cdot C_{0}}{T^{2}}\right)$, which is inversely proportional to the square of the temperature and therefore it significantly increases if the temperature falls.

\footnotetext{
${ }^{\mathrm{a}}$ Corresponding author: magda.vestfalova@tul.cz
} 
To estimate the coefficients $A_{0}, B_{0}, C_{0}$ was used IAPWS-95 data of water steam at different temperatures and such densities at which the maximum error of the model of an ideal gas from real data was $5 \%$ approximately.

If the thermodynamic properties of water vapor was calculated from the ideal gas state equation adjusted to the real behavior of water steam through the $2 \mathrm{nd}$, 4 th and 6th member in BWR equation, the area, where the error of calculated values was less than $1 \%$, significantly increased: for temperature $1000^{\circ} \mathrm{C}$ to the pressure $50 \mathrm{MPa}$, for temperature $500^{\circ} \mathrm{C}$ to the pressure $22 \mathrm{MPa}$. On the limit curve, the accuracy $1 \%$ is maintained at temperatures up to $290^{\circ} \mathrm{C}$. In Table 1 . the region is indicated by a pink color, in the Figure 1. by blue color.

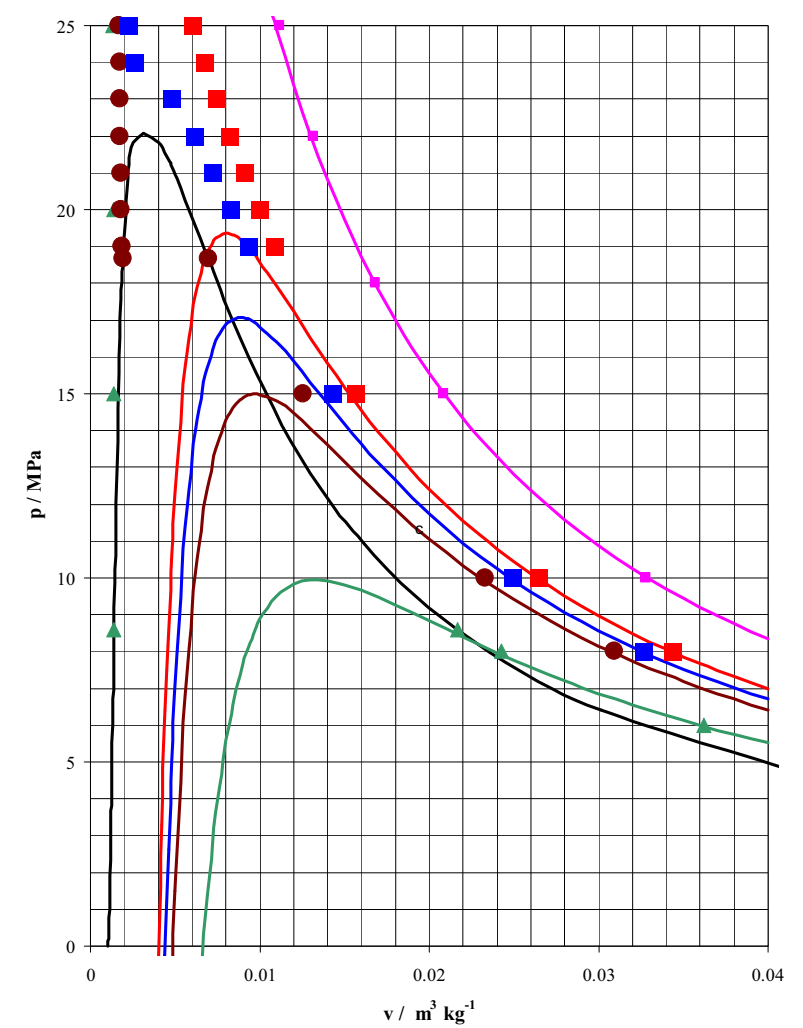

Figure 3. Comparison of isotherms of model with correction $A_{0}$, $B_{0}$ and $C_{0}$ with real isotherms: thick line - the limit curve; green - isotherm $300^{\circ} \mathrm{C}$; brown - isotherm $360^{\circ} \mathrm{C}$; bleu - isotherm $380^{\circ} \mathrm{C}$; red - isotherm $400^{\circ} \mathrm{C}$; pink - isotherm $500^{\circ} \mathrm{C}$; lines model with correction $A_{0}, B_{0}$ and $C_{0}$ isotherms; points - real values.

\section{Correction using members proportional to the third power of density}

At low values of specific volume, however, the error is still too big in gaseous state too, see Figure 3. At low specific volumes, the model gives the lower value of pressure than its real value. Obviously this is the area in which begin to apply the correcting members with higher than the square power of the density. The third and the fifth member are proportional to the third power of density. The fifth member $\left(-a \cdot \rho^{3}\right)$ is directly proportional only to the third power of density and apparently corresponds to the absolute deviation between the calculated and real isotherm. Whereas it is necessary to make such a correction that "increases" calculated pressure, in BWR equation that member will be added (negative value of $a$ ). The deviation of calculated pressures from the real values of pressure is not the same for all temperatures - the higher the temperature, the smaller the difference between the actual and the calculated pressure. The original correction (fifth member) is therefore necessary to "alleviate" (decrease) by correction using the third member $\left(T \cdot \rho^{3} \cdot b\right)$, whose influence is greater the higher the temperature of the gas. To estimate the coefficients $a$ and $b$ was used IAPWS95 data of steam water at different temperatures and such densities at which the maximum error of the model of an ideal gas with the previous correction (2nd, 4th and 7th member in a BWR equation) from the real data was approximately $5 \%$.

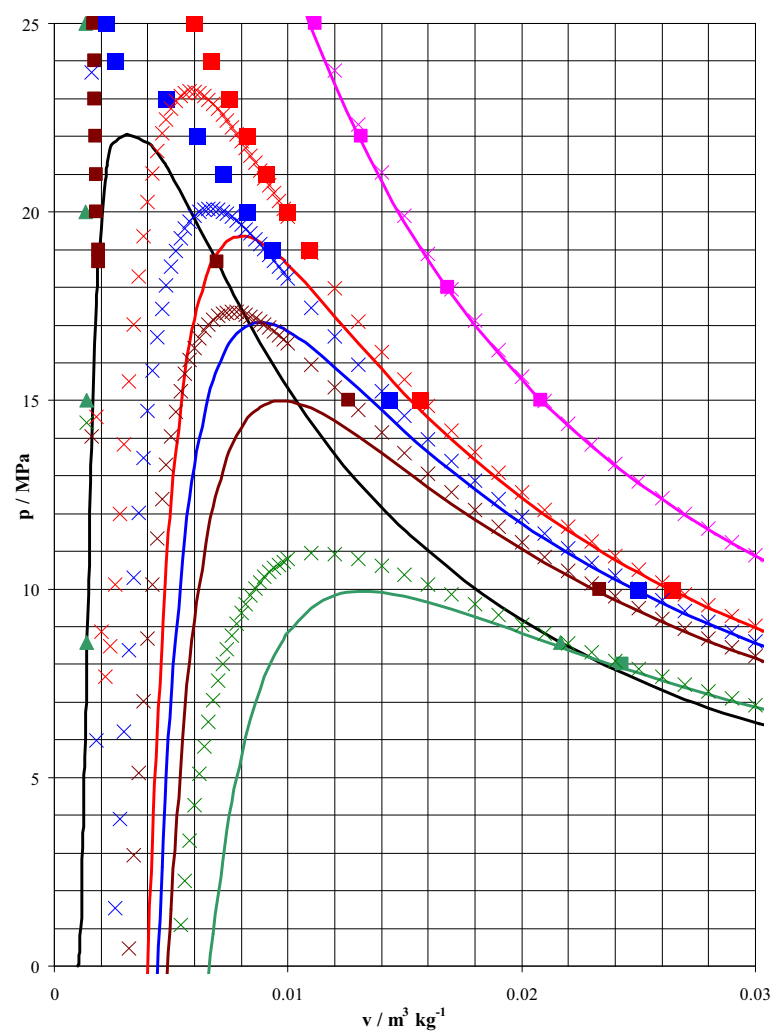

Figure 4. Comparison of isotherms of model with correction $A_{0}$ $B_{0}, C_{0}, a$ and $b$ (lines) with isotherms of model with correction only $A_{0}, B_{0}$ and $C_{0}$ (crosses) and with real isotherms (points): thick line - the limit curve; green - isotherm $300^{\circ} \mathrm{C}$; brown isotherm $360^{\circ} \mathrm{C}$; bleu - isotherm $380^{\circ} \mathrm{C}$; red - isotherm $400^{\circ} \mathrm{C}$; pink - isotherm $500^{\circ} \mathrm{C}$.

If the thermodynamic properties of water vapor was calculated from the ideal gas state equation adjusted to the real behavior of water steam through the $2 \mathrm{nd}$, $3 \mathrm{rd}$, 4th, 5th and 6th member in BWR equation, the area, where the error of calculated values was less than $1 \%$, increased again: on the limit curve, the accuracy $1 \%$ is maintained at temperatures up to $340^{\circ} \mathrm{C}$. In Table 1., the 
region is indicated by a green color, in the Figure 1. by green color too.

\section{Correction using members with special dependence on density}

Isotherms calculated using such equations have already the known shape of Van der Waals isotherm (with the local maximum and minimum in the area of wet steam), see Figure 4. The big mistake, however, still occurs near the critical point, it is at much lower levels of specific volume. Obviously, this is the area where it would be needed used further correction in the state equation.

The third power density is found in the eighth member $\left(\frac{c \cdot \rho^{3}}{T^{2}}\left(1+\gamma \cdot \rho^{2}\right) e^{-\gamma \cdot \rho^{2}}\right)$, but the dependence of this correction term on the density is more complicated. To illustrate the effect of the eighth member look at Figure 5., where dependence $\frac{\rho^{3}}{T^{2}}\left(1+\rho^{2}\right) e^{-\rho^{2}}$ on $\frac{1}{\rho}$ (it is on specific volume) is plotted for two different temperatures. It is evident, that this member neither monotonically decreases nor monotonically increases with increasing density (it is with decreasing specific volume), but its influence is reflected only in a certain range of densities. Its dependence has a marked maximum for concretely value of density. This member is inversely proportional to the square of temperature. His influence, therefore, probably will decrease significantly with increasing temperature. This member is significantly exhibit to correcting the shape of isotherms in the occurrence of local maxima and minima; it is below the limit curve (area of phase change). The constant $\gamma$ should be determined so that the region of influence of the eighth member coincides to the densities corresponds with the start and end of the phase transformation of water vapor.

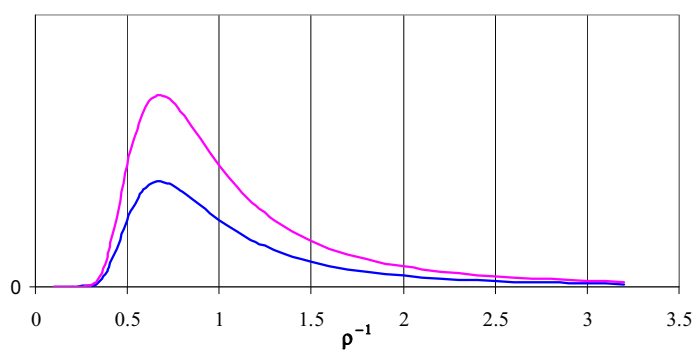

Figure 5. Dependence of $\frac{\rho^{3}}{T^{2}}\left(1+\rho^{2}\right) e^{-\rho^{2}}$ on $\frac{1}{\rho}$ for temperature $500^{\circ} \mathrm{C}$ (blue line) and for temperature $300^{\circ} \mathrm{C}$ (pink line).

The last unused member of the BWR equation is the 7 th member $-\left(a \cdot \alpha \cdot \rho^{6}\right)$. It is proportional to the sixth power of density and thus it has influence only at significantly high densities. This member does not depend on the temperature, which means that adjusts value of pressure independently on the temperature - for the some density it add to the calculated values of pressure (see the previous correction) still the same value.
When parameter $c$ and parameter $\alpha$ are estimated, we need for small values of specific volumes (where correction using the 7th member and the 8th member may have effect on the calculated value of pressure) to ensure: the applicability of the equation on the saturation line to the higher temperatures, refinement of equation near the critical point, the existence of a critical point - the inflection point on the critical isotherm, maintaining sufficient accuracy equation at high temperatures.

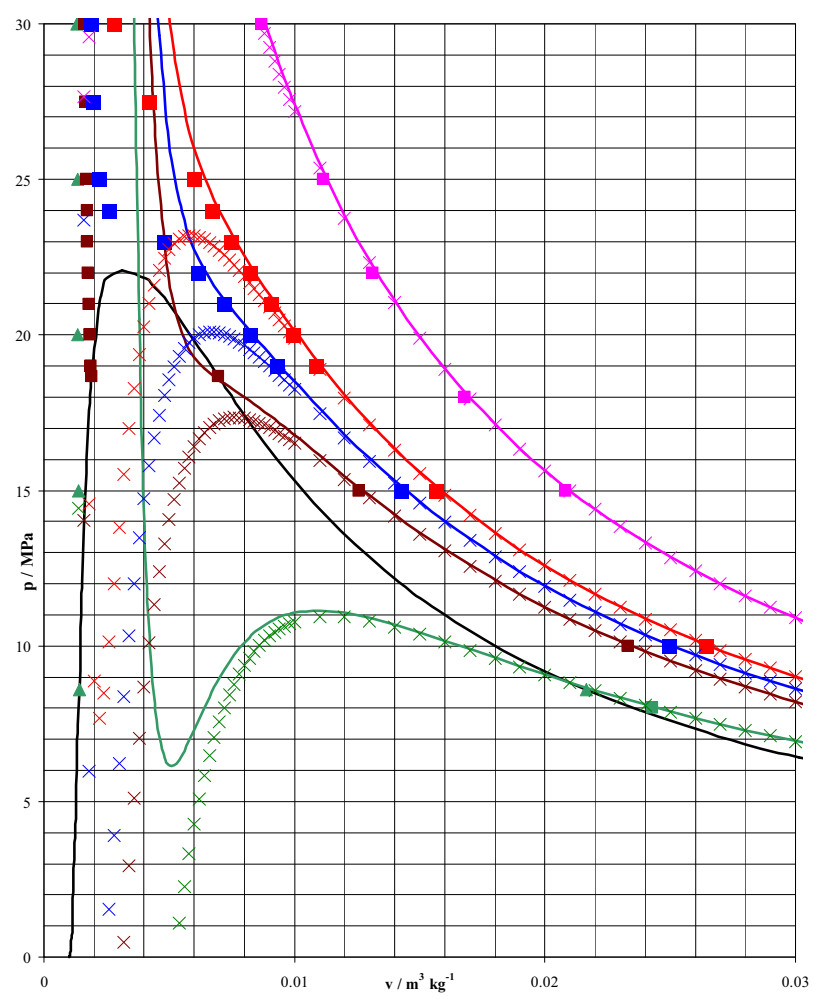

Figure 6. Comparison of isotherms of model with correction $A_{0}$, $B_{0}, C_{0}, a, b, c$ and $\alpha$ (lines) with isotherms of model with correction only $A_{0}, B_{0}, C_{0}, a$ and $b$ (crosses) and with real isotherms (points): thick line - the limit curve; green - isotherm $300^{\circ} \mathrm{C}$; brown - isotherm $360^{\circ} \mathrm{C}$; bleu - isotherm $380^{\circ} \mathrm{C}$; red isotherm $400^{\circ} \mathrm{C}$; pink - isotherm $500^{\circ} \mathrm{C}$.

It turns out that it is not possible to satisfy simultaneously these requirements for $\mathrm{H}_{2} \mathrm{O}$ using the original BWR equation. It is possible to extend the application area of the equation on the limit curve to higher temperatures, but at the cost of a reduced pressure into which an equation is usable in gas phase at high temperatures (see the yellow line in Figure 1.). Figure 6. shows some isotherms calculated by equation with correcting members with $A_{0}, B_{0}, C_{0}, a$ and $b$ (crosses) and same isotherms calculated using the BWR equation with such choice of parameters $c$ and $\alpha$, that we obtained the good accuracy on the limit curve to the high temperatures (see isotherm $360^{\circ} \mathrm{C}$ ). We see that for this temperature and pressure of saturation, BWR equation gives a more accurate value, but the shape of this isotherm is bad - it looks like supercritical isotherms (no inflection point). If we use such combination of correction parameters that on the critical isotherm (it is on the isotherm $373.946^{\circ} \mathrm{C}$ ) is an inflection point, we get the critical point shifted 
significantly to the left (to higher values of specific volumes than corresponds with data IAPWS-95) and the error of calculated values on the boundary curve is much more even for lower temperatures.

It turns out that if we want to calculate the thermodynamic properties of steam using "simple" equation of state based on the original BWR equation, it is useless to use its full form - 7th and 8th correction terms have not brought any significant refinement of calculated values of pressure. For routine engineering calculations of water vapor it is thus possible to use a simplified BWR equation of the form

$$
p=r T \rho+r T \rho^{2} B_{0}+r T \rho^{3} b-A_{0} \rho^{2}-a \rho^{3}-\frac{\rho^{2} C_{0}}{T^{2}}
$$

This equation gives sufficiently accurate results. When we use simplified BWR equation with coefficients, which we obtained by shown procedure, we can calculate the properties of water vapor to a state of beginning of condensation to the pressure $15 \mathrm{MPa}$ (temperature $340^{\circ} \mathrm{C}$ ). For high temperature, equation can be used for even higher pressures (see Figure 1.), but pressures up to $30 \mathrm{MPa}$.

\section{Conclusions}

Simplified BWR equation using coefficients derived in this paper, is less accurate than the equations used in IAPWS, near the critical point and in liquid areas even is not usable at all. The great advantage is the simplicity of equation, and thus easier possibility of calculating of the properties of the water vapor. Another advantage is the possibility of derivation of the relationship for other thermodynamic properties (isobaric specific heat capacity $c_{p}$, enthalpy $\left.h, \ldots\right)[10]$ and thus the simple possibility of calculating of the thermodynamic processes in the superheated steam without the using of thermodynamic tables of water and steam.

\section{Acknowledgment}

Work was supported by the project SGS 21000.

\section{References}

1. M.Vestfálová, P.Šafařík, Experimental Fluid Mechanics 2015 (to be published)

2. W.Wagner, A.Pruß, The IAPWS Formulation 1995 for the Thermodynamic Properties of Ordinary Water Substance for General and Scientific Use, Journal of Physical and Chemical Reference Data, 31: 387-535 (2002)

3. O.Šifner, J.Klomfar, Mezinárodni standardy termofyzikálních vlastností vody a vodni páry, (1996)

4. M. Benedict, G. Webb, L. C. Rubin, Journal of Chemical Physics 10 (12): 747-758 (1942)

5. K. E. Starling, Fluid Properties for Light Petroleum Systems, (1973)

6. R. C. Reid, J. M. Prausnitz, B. E. Poling, The Properties of Gases \& Liquids (4th ed.) (1987)
7. B. A. Younglove, J. F. Ely, Journal of Physical and Chemical Reference Data 16 (4): 577 (1987)

8. M. Benedict, G. Webb, L. C. Rubin, Chemical Engineering Progress (CEP) 47 (8): 419-422 (1951)

9. J. Novák, Termodynamické vlastnosti plynů, (2007)

10. M. Vestfálová, Experimental Fluid Mehcanics (2014) 